\title{
On new inequalities of Fejér-Hermite-Hadamard type for differentiable $(\alpha, m)$-preinvex mappings
}

\author{
Yi-Chao Zhang, Ting-Song Du*, Jiao Pan \\ Department of Mathematics, College of Science, China Three Gorges University, Yichang, 443002, China
}

*Corresponding author, e-mail: tingsongdu@ctgu.edu.cn

Received 18 Aug 2016

Accepted $16 \mathrm{Jul} 2017$

\begin{abstract}
The authors establish several new inequalities of the Fejér-Hermite-Hadamard type for mappings which have absolute values of the first derivatives which are $(\alpha, m)$-preinvex. The results presented provide extensions of some known results. These new established inequalities are also applied to construct inequalities for special means.
\end{abstract}

KEYWORDS: integral inequalities, convex mappings

MSC2010: 26A51 26D07 26D15 26D20

\section{INTRODUCTION}

Let $f: I \subseteq \mathbb{R} \rightarrow \mathbb{R}$ be a convex mapping and $a, b \in I$ with $a<b$. The inequality

$$
f\left(\frac{a+b}{2}\right) \leqslant \frac{1}{b-a} \int_{a}^{b} f(x) \mathrm{d} x \leqslant \frac{f(a)+f(b)}{2}
$$

is referred to as Hermite-Hadamard's inequality and is one of the most famous results for convex mappings. (1) ${ }^{1}$ :

Fejér provided a weighted generalization of

$$
\begin{aligned}
f\left(\frac{a+b}{2}\right) \int_{a}^{b} w(x) \mathrm{d} x & \leqslant \int_{a}^{b} f(x) w(x) \mathrm{d} x \\
& \leqslant \frac{f(a)+f(b)}{2} \int_{a}^{b} w(x) \mathrm{d} x
\end{aligned}
$$

where $f:[a, b] \rightarrow \mathbb{R}$ is a convex function and $f:$ $[a, b] \rightarrow \mathbb{R}$ is nonnegative, integrable and symmetrical about $\frac{1}{2}(a+b)$.

Definition 1 A set $S \subseteq \mathbb{R}^{n}$ is said to be an invex set with respect to the mapping $\eta: S \times S \rightarrow \mathbb{R}^{n}$ if $x+$ $t \eta(y, x) \in S$ for every $x, y \in S$ and $t \in[0,1]^{2}$.

Definition 2 The function $f$ defined on the invex set $K \subseteq \mathbb{R}^{n}$ is said to be preinvex with respect to $\eta$ if for every $x, y \in K$ and $t \in[0,1]^{2}$

$$
f(x+t \eta(y, x)) \leqslant(1-t) f(x)+t f(y) .
$$

Definition 3 The function $f$ on the invex set $K \subseteq$ $\left[0, b^{*}\right], b^{*}>0$, is said to be $(\alpha, m)$-preinvex with respect to $\eta$ if

$$
f(x+t \eta(y, x)) \leqslant\left(1-t^{\alpha}\right) f(x)+m t^{\alpha} f\left(\frac{y}{m}\right)
$$

holds for all $x, y \in K, t \in[0,1]$ and $(\alpha, m) \in(0,1] \times$ $(0,1]^{3}$.

Theorem 1 (Ref. 4) Let $f:[a, a+\eta(b, a)] \rightarrow$ $(0, \infty)$ be an open preinvex function on the interval of real numbers $K^{\circ}$ (the interior of $K$ ) and $a, b \in K^{\circ}$ with $a \leqslant a+\eta(b, a)$. Then

$$
\begin{aligned}
f\left(\frac{2 a+\eta(b, a)}{2}\right) & \leqslant \frac{1}{\eta(b, a)} \int_{a}^{a+\eta(b, a)} f(x) \mathrm{d} x \\
& \leqslant \frac{f(a)+f(b)}{2} .
\end{aligned}
$$

In Ref. 5, they found the right-sided integral inequalities of Fejér type concerning the product of an $s$-convex mapping and a symmetric function. In Ref. 6 some left-sided Fejér-Hermite-Hadamard type inequalities for preinvex mappings were also established.

In recent years, many researchers have studied bounds for both Hermite-Hadamard and Fejér type inequalities via different classes of convex mappings; for generalizations, refinements, variations and new inequalities for them, see Refs. 7-19. Based on this literature and especially the idea in Refs. 5,6, by discovering a weighted identity involving a symmetric mapping and a differentiable preinvex mapping defined on open invex subset, we 
derive the right-sided new Fejér-Hermite-Hadamard type inequalities for mappings which have absolute values of the first derivatives which are $(\alpha, m)$ preinvex. Our results give extensions of some known results. The new integral inequalities are also applied to special means.

\section{MAIN RESULTS}

Lemma 1 Let $K \subseteq \mathbb{R}$ be an open invex subset with respect to $\eta: K \times K \rightarrow \mathbb{R}$ and $a, b \in K, a<b$ with $\eta(b, a)>0$. Assume that $f: K \rightarrow \mathbb{R}$ is a differentiable mapping on $K$ such that $f^{\prime} \in L_{1}([a, a+\eta(b, a)])$. If $g:[a, a+\eta(b, a)] \rightarrow[0, \infty)$ is an integrable mapping and symmetrical about $a+\frac{1}{2} \eta(b, a)$, then

$$
\begin{gathered}
{\left[\frac{f(a)+f(a+\eta(b, a))}{2}\right] \int_{a}^{a+\eta(b, a)} g(x) \mathrm{d} x} \\
-\int_{a}^{a+\eta(b, a)} f(x) g(x) \mathrm{d} x \\
=\frac{\eta(b, a)}{4}\left\{\int_{0}^{1}\left[\int_{\psi(t)}^{\varphi(t)} g(x) \mathrm{d} x\right]\right. \\
\left.\times\left[f^{\prime}(\varphi(t))-f^{\prime}(\psi(t))\right] \mathrm{d} t\right\}
\end{gathered}
$$

where $\varphi(t)=a+\frac{1}{2} t \eta(b, a)$ and $\psi(t)=a+(1-$ $\left.\frac{1}{2} t\right) \eta(b, a)$. In particular,

$$
\begin{aligned}
& \mid \frac{f(a)+f(a+\eta(b, a))}{2} \int_{a}^{a+\eta(b, a)} g(x) \mathrm{d} x \\
& -\int_{a}^{a+\eta(b, a)} f(x) g(x) \mathrm{d} x \mid \\
& \leqslant \frac{\eta(b, a)}{4}\left\{\int_{0}^{1}\left|\left[\int_{\psi(t)}^{\varphi(t)} g(x) \mathrm{d} x\right]\right|\right. \\
& \left.\times\left[\left|f^{\prime}(\varphi(t))\right|+\left|f^{\prime}(\psi(t))\right|\right] \mathrm{d} t\right\}
\end{aligned}
$$

and

$$
\begin{gathered}
\mid \frac{f(a)+f(a+\eta(b, a))}{2} \int_{a}^{a+\eta(b, a)} g(x) \mathrm{d} x \\
-\int_{a}^{a+\eta(b, a)} f(x) g(x) \mathrm{d} x \mid \\
\leqslant \frac{\eta^{2}(b, a)}{4}\|g\|_{\infty} \\
\times \int_{0}^{1}(1-t)\left[\left|f^{\prime}(\varphi(t))\right|+\left|f^{\prime}(\psi(t))\right|\right] \mathrm{d} t
\end{gathered}
$$

where $\|g\|_{\infty}=\sup _{t \in[a, a+\eta(b, a)]} g(t)$.
Proof: Since $g(x)$ is symmetrical about $a+\frac{1}{2} \eta(b, a)$, $g(\psi(t))=g(\varphi(t))$ for all $t \in[0,1]$. Hence

$$
\begin{aligned}
I^{*}= & \frac{\eta(b, a)}{4} \int_{0}^{1}\left[\int_{\psi(t)}^{\varphi(t)} g(x) \mathrm{d} x\right] \\
& \times\left[f^{\prime}(\varphi(t))-f^{\prime}(\psi(t))\right] \mathrm{d} t \\
= & \frac{\eta(b, a)}{4} \int_{0}^{1}\left[\int_{\psi(t)}^{\varphi(t)} g(x) \mathrm{d} x\right] f^{\prime}(\varphi(t)) \mathrm{d} t \\
& \quad-\frac{\eta(b, a)}{4} \int_{0}^{1}\left[\int_{\psi(t)}^{\varphi(t)} g(x) \mathrm{d} x\right] f^{\prime}(\psi(t)) \mathrm{d} t \\
:= & I_{1}-I_{2} .
\end{aligned}
$$

Via integration by parts we obtain

$$
\begin{aligned}
I_{1}= & \frac{\eta(b, a)}{4} \int_{0}^{1}\left[\int_{\psi(t)}^{\varphi(t)} g(x) \mathrm{d} x\right] f^{\prime}(\varphi(t)) \mathrm{d} t \\
= & \frac{1}{2} \int_{0}^{1}\left[\int_{\psi(t)}^{\varphi(t)} g(x) \mathrm{d} x\right] \mathrm{d}[f(\varphi(t))] \\
= & \frac{1}{2}\left\{\left.\left[\int_{\psi(t)}^{\varphi(t)} g(x) \mathrm{d} x\right] f(\varphi(t))\right|_{0} ^{1}\right. \\
& \left.-\frac{\eta(b, a)}{2} \int_{0}^{1}[g(\varphi(t))+g(\psi(t))] f(\varphi(t)) \mathrm{d} t\right\} \\
= & \frac{1}{2}\left\{f(a) \int_{a}^{a+\eta(b, a)} g(x) \mathrm{d} x\right. \\
& \left.-\eta(b, a) \int_{0}^{1} g(\varphi(t)) f(\varphi(t)) \mathrm{d} t\right\} \\
= & \frac{1}{2}\left\{f(a) \int_{a}^{a+\eta(b, a)} g(x) \mathrm{d} x\right. \\
& \left.-2 \int_{a}^{a+(1 / 2) \eta(b, a)} g(x) f(x) \mathrm{d} x\right\}
\end{aligned}
$$

and similarly,

$$
\begin{aligned}
I_{2}=-\frac{1}{2}\left\{f(a+\eta(b, a)) \int_{a}^{a+\eta(b, a)} g(x) \mathrm{d} x\right. \\
\left.-2 \int_{a+(1 / 2) \eta(b, a)}^{a+\eta(b, a)} g(x) f(x) \mathrm{d} x\right\} .
\end{aligned}
$$

From $I_{1}$ and $I_{2}$, it follows that

$$
I^{*}=I_{1}-I_{2}
$$




$$
\begin{aligned}
=[ & \left.\frac{f(a)+f(a+\eta(b, a))}{2}\right] \int_{a}^{a+\eta(b, a)} g(x) \mathrm{d} x \\
& -\int_{a}^{a+\eta(b, a)} f(x) g(x) \mathrm{d} x
\end{aligned}
$$

which is the required result (6). Using Minkowski's inequality, it is straightforward to obtain (7) and (8).

Remark 1 If $g(x)=1, x \in[a, a+\eta(b, a)]$ in Lemma 1, then (6) reduces to

$$
\begin{array}{r}
\frac{f(a)+f(a+\eta(b, a))}{2}-\frac{1}{\eta(b, a)} \int_{a}^{a+\eta(b, a)} f(x) \mathrm{d} x \\
=\frac{\eta(b, a)}{4} \int_{0}^{1}(t-1)\left[f^{\prime}\left(a+\frac{t}{2} \eta(b, a)\right)\right. \\
\left.-f^{\prime}\left(a+\left(1-\frac{t}{2}\right) \eta(b, a)\right)\right] \mathrm{d} t .
\end{array}
$$

Remark 2 If $\eta(b, a)=b-a$ in Lemma 1, then (6) becomes

$$
\begin{aligned}
& {\left[\frac{f(a)+f(b)}{2}\right] \int_{a}^{b} g(x) \mathrm{d} x-\int_{a}^{b} f(x) g(x) \mathrm{d} x} \\
& =\frac{b-a}{4}\left\{\int _ { 0 } ^ { 1 } [ \int _ { \psi ( t ) } ^ { \varphi ( t ) } g ( x ) \mathrm { d } x ] \left[f^{\prime}\left(\frac{2-t}{2} a+\frac{t}{2} b\right)\right.\right. \\
& \left.\left.\quad-f^{\prime}\left(\frac{t}{2} a+\frac{2-t}{2} b\right)\right] \mathrm{d} t\right\} \\
& =\frac{b-a}{4}\left\{\int_{0}^{1}\left[\int_{\psi(t)}^{\varphi(t)} g(x) \mathrm{d} x\right]\right. \\
& \quad \times\left[f^{\prime}\left((1-t) a+t \frac{a+b}{2}\right)\right. \\
& \left.\left.\quad-f^{\prime}\left(t \frac{a+b}{2}+(1-t) b\right)\right] \mathrm{d} t\right\}
\end{aligned}
$$

where $\varphi(t)=(1-t) a+\frac{1}{2} t(a+b)$ and $\psi(t)=\frac{1}{2} t(a+$ b) $+(1-t) b$.

The second integral identity in (10) is proved in Ref. 5 [page 754, Lemma 2.1].

With the help of Lemma 1, a new upper bound for the right-hand side of (2) via $(\alpha, m)$-preinvex mappings is shown with the following inequality.

Theorem 2 Let $K \subseteq \mathbb{R}_{0}$ be an open invex subset with respect to $\eta: K \times K \rightarrow \mathbb{R}, a, b \in K$ and $0 \leqslant a<b$ with $\eta(b, a)>0$. Suppose $f: K \rightarrow \mathbb{R}_{0}$ is a differentiable mapping on $K$ such that $f^{\prime} \in L_{1}([a, a+\eta(b, a)])$ and $g:[a, a+\eta(b, a)] \rightarrow[0, \infty)$ is an integrable mapping and symmetrical about $a+\frac{1}{2} \eta(b, a)$. If $\left|f^{\prime}\right|^{q}$ for $q \geqslant 1$ is $(\alpha, m)$-preinvex on $[a, b / m], \alpha \in(0,1]$ and $m \in$ $(0,1]$ with $(b / m) \in K$, then

$$
\begin{gathered}
\mid \frac{f(a)+f(a+\eta(b, a))}{2} \int_{a}^{a+\eta(b, a)} g(x) \mathrm{d} x \\
\quad-\int_{a}^{a+\eta(b, a)} f(x) g(x) \mathrm{d} x \mid \\
\leqslant \frac{\eta^{2}(b, a)}{4}\left(\frac{1}{2}\right)^{1-1 / q}\|g\|_{\infty} \\
\times\left\{\left[\left(\frac{1}{2}-\frac{1}{2^{\alpha}(\alpha+1)(\alpha+2)}\right)\left|f^{\prime}(a)\right|^{q}\right.\right. \\
\left.+\frac{m}{2^{\alpha}(\alpha+1)(\alpha+2)}\left|f^{\prime}\left(\frac{b}{m}\right)\right|^{q}\right]^{1 / q} \\
+\left[\left(\frac{1}{2}-\frac{1+\alpha 2^{\alpha+1}}{2^{\alpha}(\alpha+1)(\alpha+2)}\right)\left|f^{\prime}(a)\right|^{q}\right. \\
\left.\left.+\frac{m\left(1+\alpha 2^{\alpha+1}\right)}{2^{\alpha}(\alpha+1)(\alpha+2)}\left|f^{\prime}\left(\frac{b}{m}\right)\right|^{q}\right]^{1 / q}\right\} .
\end{gathered}
$$

Proof: By (8) in Lemma 1 and the power mean inequality, we have

$$
\begin{aligned}
& \mid \frac{f(a)+f(a+\eta(b, a))}{2} \int_{a}^{a+\eta(b, a)} g(x) \mathrm{d} x \\
& -\int_{a}^{a+\eta(b, a)} f(x) g(x) \mathrm{d} x \mid \\
& \leqslant \frac{\eta^{2}(b, a)}{4}\|g\|_{\infty}\left(\int_{0}^{1}(1-t) \mathrm{d} t\right)^{1-1 / q} \\
& \times\left\{\left[\int_{0}^{1}(1-t)\left|f^{\prime}\left(a+\frac{t}{2} \eta(b, a)\right)\right|^{q} \mathrm{~d} t\right]^{1 / q}\right. \\
& \left.+\left[\int_{0}^{1}(1-t)\left|f^{\prime}\left(a+\left(1-\frac{t}{2}\right) \eta(b, a)\right)\right|^{q} \mathrm{~d} t\right]^{1 / q}\right\} \\
& =\frac{\eta^{2}(b, a)}{4}\|g\|_{\infty}\left(\frac{1}{2}\right)^{1-1 / q} \\
& \times\left\{\left[\int_{0}^{1}(1-t)\left|f^{\prime}\left(a+\frac{t}{2} \eta(b, a)\right)\right|^{q} \mathrm{~d} t\right]^{1 / q}\right. \\
& \left.+\left[\int_{0}^{1}(1-t)\left|f^{\prime}\left(a+\left(1-\frac{t}{2}\right) \eta(b, a)\right)\right|^{q} \mathrm{~d} t\right]^{1 / q}\right\} .
\end{aligned}
$$

Since $\left|f^{\prime}\right|^{q}$ is $(\alpha, m)$-preinvex in the second sense on $[a, b / m]$, for any $t \in[0,1]$, we have

$$
\int_{0}^{1}(1-t)\left|f^{\prime}\left(a+\frac{t}{2} \eta(b, a)\right)\right|^{q} \mathrm{~d} t
$$




$$
\begin{array}{r}
\leqslant\left|f^{\prime}(a)\right|^{q} \int_{0}^{1}(1-t)\left(1-\frac{t^{\alpha}}{2^{\alpha}}\right) \mathrm{d} t \\
+\left|f^{\prime}\left(\frac{b}{m}\right)\right|^{q} \int_{0}^{1} m(1-t) \frac{t^{\alpha}}{2^{\alpha}} \mathrm{d} t \\
=\left(\frac{1}{2}-\frac{1}{2^{\alpha}(\alpha+1)(\alpha+2)}\right)\left|f^{\prime}(a)\right|^{q} \\
\quad+\frac{m}{2^{\alpha}(\alpha+1)(\alpha+2)}\left|f^{\prime}\left(\frac{b}{m}\right)\right|^{q}
\end{array}
$$

and

$$
\begin{gathered}
\int_{0}^{1}(1-t)\left|f^{\prime}\left(a+\left(1-\frac{t}{2}\right) \eta(b, a)\right)\right|^{q} \mathrm{~d} t \\
\leqslant\left|f^{\prime}(a)\right|^{q} \int_{0}^{1}(1-t)\left[1-\left(1-\frac{t}{2}\right)^{\alpha}\right] \mathrm{d} t \\
\quad+\left|f^{\prime}\left(\frac{b}{m}\right)\right|^{q} \int_{0}^{1} m(1-t)\left(1-\frac{t}{2}\right)^{\alpha} \mathrm{d} t \\
=\left(\frac{1}{2}-\frac{1+\alpha 2^{\alpha+1}}{2^{\alpha}(\alpha+1)(\alpha+2)}\right)\left|f^{\prime}(a)\right|^{q} \\
\quad+\frac{m\left(1+\alpha 2^{\alpha+1}\right)}{2^{\alpha}(\alpha+1)(\alpha+2)}\left|f^{\prime}\left(\frac{b}{m}\right)\right|^{q} .
\end{gathered}
$$

Using (13) and (14) in (12), we deduce the required inequality (11).

Corollary 1 If $q=1$ in Theorem 2, we obtain

$$
\begin{aligned}
& \mid \frac{f(a)+f(a+\eta(b, a))}{2} \int_{a}^{a+\eta(b, a)} g(x) \mathrm{d} x \\
& \quad-\int_{a}^{a+\eta(b, a)} f(x) g(x) \mathrm{d} x \mid \\
& \leqslant \frac{\eta^{2}(b, a)}{4}\|g\|_{\infty}\left[\left(1-\frac{1+\alpha 2^{\alpha}}{2^{\alpha-1}(\alpha+1)(\alpha+2)}\right)\left|f^{\prime}(a)\right|\right. \\
& \left.\quad+\frac{m\left(1+\alpha 2^{\alpha}\right)}{2^{\alpha-1}(\alpha+1)(\alpha+2)}\left|f^{\prime}\left(\frac{b}{m}\right)\right|\right] . \quad \text { (15) }
\end{aligned}
$$

Corollary 2 If $g(x)=1$ in Theorem 2, we obtain

$$
\begin{aligned}
& \left|\frac{f(a)+f(a+\eta(b, a))}{2}-\frac{1}{\eta(b, a)} \int_{a}^{a+\eta(b, a)} f(x) \mathrm{d} x\right| \\
& \leqslant \frac{\eta(b, a)}{4}\left(\frac{1}{2}\right)^{1-1 / q}\left\{\left[\left(\frac{1}{2}-\frac{1}{2^{\alpha}(\alpha+1)(\alpha+2)}\right)\left|f^{\prime}(a)\right|^{q}\right.\right. \\
& \left.+\frac{m}{2^{\alpha}(\alpha+1)(\alpha+2)}\left|f^{\prime}\left(\frac{b}{m}\right)\right|^{q}\right]^{1 / q} \\
& +\left[\left(\frac{1}{2}-\frac{1+\alpha 2^{\alpha+1}}{2^{\alpha}(\alpha+1)(\alpha+2)}\right)\left|f^{\prime}(a)\right|^{q}\right. \\
& \left.\left.+\frac{m\left(1+\alpha 2^{\alpha+1}\right)}{2^{\alpha}(\alpha+1)(\alpha+2)}\left|f^{\prime}\left(\frac{b}{m}\right)\right|^{q}\right]^{1 / q}\right\} .
\end{aligned}
$$

Corollary 3 With the same assumptions given in Theorem 2 , if $\left|f^{\prime}(x)\right| \leqslant \Upsilon$ on $[a, a+\eta(b, a)]$ with $m=1$, we deduce

$$
\begin{aligned}
& \mid \frac{f(a)+f(a+\eta(b, a))}{2} \int_{a}^{a+\eta(b, a)} g(x) \mathrm{d} x \\
& -\int_{a}^{a+\eta(b, a)} f(x) g(x) \mathrm{d} x \mid \leqslant \frac{\eta^{2}(b, a)}{4} \Upsilon\|g\|_{\infty} .
\end{aligned}
$$

Corollary 4 If $q=1, \alpha=1, m=1$, and $g(x)=1$ in Theorem 2, we obtain

$$
\begin{array}{r}
\left|\frac{f(a)+f(a+\eta(b, a))}{2}-\frac{1}{\eta(b, a)} \int_{a}^{a+\eta(b, a)} f(x) \mathrm{d} x\right| \\
\leqslant \frac{1}{8} \eta(b, a)\left(\left|f^{\prime}(a)\right|+\left|f^{\prime}(b)\right|\right)
\end{array}
$$

which is Theorem 2.1 from [Ref. 20 page 3].

On the basis of Lemma 1 and by using Hölder's inequality, we obtain the result below.

Theorem 3 Under the conditions of Theorem 2, if $\left|f^{\prime}\right|^{q}$ for $q>1$ is $(\alpha, m)$-preinvex on $[a, b / m], \alpha \in$ $(0,1]$ and $m \in(0,1]$ with $(b / m) \in K$, then

$$
\begin{aligned}
& \mid \frac{f(a)+f(a+\eta(b, a))}{2} \int_{a}^{a+\eta(b, a)} g(x) \mathrm{d} x \\
& -\int_{a}^{a+\eta(b, a)} f(x) g(x) \mathrm{d} x \mid \\
& \leqslant \frac{\eta^{2}(b, a)}{4}\left(\frac{q-1}{2 q-1}\right)^{(q-1) / q}\|g\|_{\infty} \\
& \times\left\{\left[\left(1-\frac{1}{2^{\alpha}(\alpha+1)}\right)\left|f^{\prime}(a)\right|^{q}\right.\right. \\
& \left.+\frac{m}{2^{\alpha}(\alpha+1)}\left|f^{\prime}\left(\frac{b}{m}\right)\right|^{q}\right]^{1 / q} \\
& +\left[\left(1+\frac{1-2^{\alpha+1}}{2^{\alpha}(\alpha+1)}\right)\left|f^{\prime}(a)\right|^{q}\right. \\
& \left.\left.+\frac{m\left(2^{\alpha+1}-1\right)}{2^{\alpha}(\alpha+1)}\left|f^{\prime}\left(\frac{b}{m}\right)\right|^{q}\right]^{1 / q}\right\} .
\end{aligned}
$$

Proof: By (8) in Lemma 1 and Hölder's inequality, we have

$$
\begin{aligned}
\mid \frac{f(a)+f(a+\eta(b, a))}{2} \int_{a}^{a+\eta(b, a)} & g(x) \mathrm{d} x \\
& -\int_{a}^{a+\eta(b, a)} f(x) g(x) \mathrm{d} x \mid
\end{aligned}
$$




$$
\begin{aligned}
\leqslant \frac{\eta^{2}(b, a)}{4}\left(\int_{0}^{1}(1-t)^{q /(q-1)} \mathrm{d} t\right)^{1-1 / q}\|g\|_{\infty} \\
\times\left\{\left[\int_{0}^{1}\left|f^{\prime}\left(a+\frac{t}{2} \eta(b, a)\right)\right|^{q} \mathrm{~d} t\right]^{1 / q}\right. \\
\left.+\left[\int_{0}^{1}\left|f^{\prime}\left(a+\left(1-\frac{t}{2}\right) \eta(b, a)\right)\right|^{q} \mathrm{~d} t\right]^{1 / q}\right\} \\
=\frac{\eta^{2}(b, a)}{4}\left(\frac{q-1}{2 q-1}\right)^{(q-1) / q}\|g\|_{\infty} \\
\times\left\{\left[\int_{0}^{1}\left|f^{\prime}\left(a+\frac{t}{2} \eta(b, a)\right)\right|^{q} \mathrm{~d} t\right]^{1 / q}\right. \\
\left.+\left[\int_{0}^{1}\left|f^{\prime}\left(a+\left(1-\frac{t}{2}\right) \eta(b, a)\right)\right|^{q} \mathrm{~d} t\right]^{1 / q}\right\} .
\end{aligned}
$$

Since $\left|f^{\prime}\right|^{q}$ is $(\alpha, m)$-preinvex in the second sense on $[a, b / m]$, for any $t \in[0,1]$, we have

$$
\begin{aligned}
& \int_{0}^{1}\left|f^{\prime}\left(a+\frac{t}{2} \eta(b, a)\right)\right|^{q} \mathrm{~d} t \\
& \leqslant\left|f^{\prime}(a)\right|^{q} \int_{0}^{1}\left(1-\frac{t^{\alpha}}{2^{\alpha}}\right) \mathrm{d} t+\left.\left|f^{\prime}\left(\frac{b}{m}\right)\right|\right|_{0} ^{q} m \frac{t^{\alpha}}{2^{\alpha}} \mathrm{d} t \\
& =\left(1-\frac{1}{2^{\alpha}(\alpha+1)}\right)\left|f^{\prime}(a)\right|^{q}+\frac{m}{2^{\alpha}(\alpha+1)}\left|f^{\prime}\left(\frac{b}{m}\right)\right|^{q}
\end{aligned}
$$

and

$$
\begin{gathered}
\int_{0}^{1}\left|f^{\prime}\left(a+\left(1-\frac{t}{2}\right) \eta(b, a)\right)\right|^{q} \mathrm{~d} t \\
\leqslant\left|f^{\prime}(a)\right|^{q} \int_{0}^{1}\left[1-\left(1-\frac{t}{2}\right)^{\alpha}\right] \mathrm{d} t \\
+\left|f^{\prime}\left(\frac{b}{m}\right)\right|^{q} \int_{0}^{1} m\left(1-\frac{t}{2}\right)^{\alpha} \mathrm{d} t \\
=\left(1+\frac{1-2^{\alpha+1}}{2^{\alpha}(\alpha+1)}\right)\left|f^{\prime}(a)\right|^{q} \\
+\frac{m\left(2^{\alpha+1}-1\right)}{2^{\alpha}(\alpha+1)}\left|f^{\prime}\left(\frac{b}{m}\right)\right|^{q} .
\end{gathered}
$$

Using (21), (22) in (20), we deduce the result (19).

Corollary 5 If $g(x)=1$ with $\alpha=m=1$ in Theorem 3 , we obtain

$$
\left|\frac{f(a)+f(a+\eta(b, a))}{2}-\frac{1}{\eta(b, a)} \int_{a}^{a+\eta(b, a)} f(x) \mathrm{d} x\right|
$$

$$
\begin{aligned}
\leqslant & \frac{\eta(b, a)}{4}\left(\frac{q-1}{2 q-1}\right)^{(q-1) / q} \\
& \times\left\{\left[\frac{3}{4}\left|f^{\prime}(a)\right|^{q}+\frac{1}{4}\left|f^{\prime}(b)\right|^{q}\right]^{1 / q}\right. \\
& \left.+\left[\frac{1}{4}\left|f^{\prime}(a)\right|^{q}+\frac{3}{4}\left|f^{\prime}(b)\right|^{q}\right]^{1 / q}\right\} \\
\leqslant & \frac{\eta(b, a)}{4}\left(\frac{q-1}{2 q-1}\right)^{\frac{q-1}{q}} \frac{3^{1 / q}+1}{4^{1 / q}}\left(\left|f^{\prime}(a)\right|+\left|f^{\prime}(b)\right|\right) .
\end{aligned}
$$

Here, $0<1 / q<1$ for $q>1$. To prove the second inequality above, we use the fact that $\sum_{i=1}^{\mathrm{n}}\left(a_{i}+\right.$ $\left.b_{i}\right)^{r} \leqslant \sum_{i=1}^{\mathrm{n}} a_{i}^{r}+\sum_{i=1}^{\mathrm{n}} b_{i}^{r}$, for $0 \leqslant r<1, a_{1}, \ldots, a_{\mathrm{n}} \geqslant$ 0 and $b_{1}, \ldots, b_{\mathrm{n}} \geqslant 0$.

Corollary 6 With the same assumptions given in Theorem 3 , if $\left|f^{\prime}(x)\right| \leqslant \Upsilon$ on $[a, a+\eta(b, a)]$ with $m=1$, we deduce

$$
\begin{gathered}
\mid \frac{f(a)+f(a+\eta(b, a))}{2} \int_{a}^{a+\eta(b, a)} g(x) \mathrm{d} x \\
\quad-\int_{a}^{a+\eta(b, a)} f(x) g(x) \mathrm{d} x \mid \\
\leqslant \frac{\eta^{2}(b, a)}{2}\left(\frac{q-1}{2 q-1}\right)^{(q-1) / q} \Upsilon\|g\|_{\infty} .
\end{gathered}
$$

Theorem 4 Let $K \subseteq \mathbb{R}_{0}$ be an open invex subset with respect to $\eta: K \times K \rightarrow \mathbb{R}, a, b \in K$ and $0 \leqslant a<b$ with $\eta(b, a)>0$. Suppose $f: K \rightarrow \mathbb{R}_{0}$ is a differentiable mapping on $K$ such that $f^{\prime} \in L_{1}([a, a+\eta(b, a)])$ and $g:[a, a+\eta(b, a)] \rightarrow[0, \infty)$ is an integrable mapping and symmetrical about $a+\frac{1}{2} \eta(b, a)$. If $\left|f^{\prime}\right|^{q}$ is $(\alpha, m)$ preinvex on $[a, b / m]$ with $q=p /(p-1), p>1, \alpha \in$ $(0,1], m \in(0,1]$ and $(b / m) \in K$, then

$$
\begin{aligned}
& \mid \frac{f(a)+f(a+\eta(b, a))}{2} \int_{a}^{a+\eta(b, a)} g(x) \mathrm{d} x \\
& -\int_{a}^{a+\eta(b, a)} f(x) g(x) \mathrm{d} x \mid \\
& \leqslant \frac{\eta^{2}(b, a)}{4}\left(\frac{1}{p+1}\right)^{1 / p}\|g\|_{\infty} \\
& \times\left\{\left[\left(1-\frac{1}{2^{\alpha}(\alpha+1)}\right)\left|f^{\prime}(a)\right|^{q}\right.\right. \\
& \left.+\frac{m}{2^{\alpha}(\alpha+1)}\left|f^{\prime}\left(\frac{b}{m}\right)\right|^{q}\right]^{1 / q} \\
& +\left[\left(1+\frac{1-2^{\alpha+1}}{2^{\alpha}(\alpha+1)}\right)\left|f^{\prime}(a)\right|^{q}\right.
\end{aligned}
$$




$$
\left.\left.+\frac{m\left(2^{\alpha+1}-1\right)}{2^{\alpha}(\alpha+1)}\left|f^{\prime}\left(\frac{b}{m}\right)\right|^{q}\right]^{1 / q}\right\} .
$$

Proof: By (8) in Lemma 1 and Hölder's inequality for $p>1$, we obtain

$$
\begin{aligned}
& \mid \frac{f(a)+f(a+\eta(b, a))}{2} \int_{a}^{a+\eta(b, a)} g(x) \mathrm{d} x \\
& -\int_{a}^{a+\eta(b, a)} f(x) g(x) \mathrm{d} x \mid \\
& \leqslant \frac{\eta^{2}(b, a)}{4}\|g\|_{\infty}\left(\int_{0}^{1}(1-t)^{p} \mathrm{~d} t\right)^{1 / p} \\
& \times\left\{\left[\int_{0}^{1}\left|f^{\prime}\left(a+\frac{t}{2} \eta(b, a)\right)\right|^{q} \mathrm{~d} t\right]^{1 / q}\right. \\
& \left.+\left[\int_{0}^{1}\left|f^{\prime}\left(a+\left(1-\frac{t}{2}\right) \eta(b, a)\right)\right|^{q} \mathrm{~d} t\right]^{1 / q}\right\} \\
& =\frac{\eta^{2}(b, a)}{4}\|g\|_{\infty}\left(\frac{1}{p+1}\right)^{1 / p} \\
& \times\left\{\left[\int_{0}^{1}\left|f^{\prime}\left(a+\frac{t}{2} \eta(b, a)\right)\right|^{q} \mathrm{~d} t\right]^{1 / q}\right. \\
& \left.+\left[\int_{0}^{1}\left|f^{\prime}\left(a+\left(1-\frac{t}{2}\right) \eta(b, a)\right)\right|^{q} \mathrm{~d} t\right]^{1 / q}\right\} .
\end{aligned}
$$

Using (21) and (22) in (26), we obtain the result (25).

Corollary 7 If taking $g(x)=1$ with $\alpha=m=1$ in Theorem 4, we obtain

$$
\begin{aligned}
& \left|\frac{f(a)+f(a+\eta(b, a))}{2}-\frac{1}{\eta(b, a)} \int_{a}^{a+\eta(b, a)} f(x) \mathrm{d} x\right| \\
& \leqslant \frac{\eta(b, a)}{4}\left(\frac{1}{p+1}\right)^{1 / p}\left\{\left[\frac{3}{4}\left|f^{\prime}(a)\right|^{q}+\frac{1}{4}\left|f^{\prime}(b)\right|^{q}\right]^{1 / q}\right. \\
& \left.\quad+\left[\frac{1}{4}\left|f^{\prime}(a)\right|^{q}+\frac{3}{4}\left|f^{\prime}(b)\right|^{q}\right]^{1 / q}\right\} \\
& \leqslant \frac{\eta(b, a)}{4}\left(\frac{1}{p+1}\right)^{1 / p} \frac{3^{1 / q}+1}{4^{1 / q}}\left(\left|f^{\prime}(a)\right|+\left|f^{\prime}(b)\right|\right)
\end{aligned}
$$

where $p^{-1}+q^{-1}=1$. To prove the second inequality above, we use the same method as in Corollary 5.

Corollary 8 With the same assumptions given in Theorem 4 , if $\left|f^{\prime}(x)\right| \leqslant \Upsilon$ on $[a, a+\eta(b, a)]$ with $m=1$, we obtain

$$
\begin{gathered}
\mid \frac{f(a)+f(a+\eta(b, a))}{2} \int_{a}^{a+\eta(b, a)} g(x) \mathrm{d} x \\
\quad-\int_{a}^{a+\eta(b, a)} f(x) g(x) \mathrm{d} x \mid \\
\leqslant \frac{\eta^{2}(b, a)}{2}\left(\frac{1}{p+1}\right)^{1 / p} \Upsilon\|g\|_{\infty},
\end{gathered}
$$

where $p^{-1}+q^{-1}=1$.

Theorem 5 Suppose that all the assumptions of Theorem 4 are satisfied. Then

$$
\begin{gathered}
\mid \frac{f(a)+f(a+\eta(b, a))}{2} \int_{a}^{a+\eta(b, a)} g(x) \mathrm{d} x \\
-\int_{a}^{a+\eta(b, a)} f(x) g(x) \mathrm{d} x \mid \\
\leqslant \frac{\eta^{2}(b, a)}{4}\left(\frac{q-1}{2 q-p-1}\right)^{(q-1) / q}\|g\|_{\infty} \\
\times\left\{\left[\left(\frac{1}{p+1}-\frac{1}{2^{\alpha}} \beta(\alpha+1, p+1)\right)\left|f^{\prime}(a)\right|^{q}\right.\right. \\
\left.+\quad+\frac{m}{2^{\alpha}} \beta(\alpha+1, p+1)\left|f^{\prime}\left(\frac{b}{m}\right)\right|^{q}\right]^{1 / q} \\
+\left[\left(\frac{1}{p+1}+\frac{(-1)^{q}}{2^{\alpha}} \beta(\alpha+1, p+1)\right)\left|f^{\prime}(a)\right|^{q}\right. \\
\left.\left.-\frac{m(-1)^{q}}{2^{\alpha}} \beta(\alpha+1, p+1)\left|f^{\prime}\left(\frac{b}{m}\right)\right|^{q}\right]^{1 / q}\right\}
\end{gathered}
$$

where

$$
\beta(x, y)=\int_{0}^{1} t^{x-1}(1-t)^{y-1} \mathrm{~d} t, \quad \forall x, y>0 .
$$

Proof: By (8) in Lemma 1 and Hölder's inequality for $p>1$, we obtain

$$
\begin{aligned}
& \mid \frac{f(a)+f(a+\eta(b, a))}{2} \int_{a}^{a+\eta(b, a)} g(x) \mathrm{d} x \\
& \quad-\int_{a}^{a+\eta(b, a)} f(x) g(x) \mathrm{d} x \mid \\
& \leqslant \frac{\eta^{2}(b, a)}{4}\|g\|_{\infty}\left(\int_{0}^{1}(1-t)^{(q-p) /(q-1)} \mathrm{d} t\right)^{(q-1) / q} \\
& \quad \times\left\{\left[\int_{0}^{1}(1-t)^{p}\left|f^{\prime}\left(a+\frac{t}{2} \eta(b, a)\right)\right|^{q} \mathrm{~d} t\right]^{1 / q}\right. \\
& \left.+\left[\int_{0}^{1}(1-t)^{p}\left|f^{\prime}\left(a+\left(1-\frac{t}{2}\right) \eta(b, a)\right)\right|^{q} \mathrm{~d} t\right]^{1 / q}\right\}
\end{aligned}
$$




$$
\begin{aligned}
= & \frac{\eta^{2}(b, a)}{4}\|g\|_{\infty}\left(\frac{q-1}{2 q-p-1}\right)^{(q-1) / q} \\
& \times\left\{\left[\int_{0}^{1}(1-t)^{p}\left|f^{\prime}\left(a+\frac{t}{2} \eta(b, a)\right)\right|^{q} \mathrm{~d} t\right]^{1 / q}\right. \\
& \left.+\left[\left.\int_{0}^{1}(1-t)^{p}\left|f^{\prime}\left(a+\left(1-\frac{t}{2}\right) \eta(b, a)\right)\right|\right|^{q} \mathrm{~d} t\right]^{1 / q}\right\} .
\end{aligned}
$$

Since $\left|f^{\prime}\right|^{q}$ is $(\alpha, m)$-preinvex in the second sense on $[a, b / m]$, for any $t \in[0,1]$, we have

$$
\begin{gathered}
\int_{0}^{1}(1-t)^{p}\left|f^{\prime}\left(a+\frac{t}{2} \eta(b, a)\right)\right|^{q} \mathrm{~d} t \\
\leqslant\left|f^{\prime}(a)\right| \int_{0}^{q}(1-t)^{p}\left(1-\frac{t^{\alpha}}{2^{\alpha}}\right) \mathrm{d} t \\
\quad+\left|f^{\prime}\left(\frac{b}{m}\right)\right|^{q} \int_{0}^{1} m(1-t)^{p} \frac{t^{\alpha}}{2^{\alpha}} \mathrm{d} t \\
=\left(\frac{1}{p+1}-\frac{1}{2^{\alpha}} \beta(\alpha+1, p+1)\right)\left|f^{\prime}(a)\right|^{q} \\
\quad+\frac{m}{2^{\alpha}} \beta(\alpha+1, p+1)\left|f^{\prime}\left(\frac{b}{m}\right)\right|^{q}
\end{gathered}
$$

and

$$
\begin{array}{r}
\int_{0}^{1}(1-t)^{p}\left|f^{\prime}\left(a+\left(1-\frac{t}{2}\right) \eta(b, a)\right)\right|^{q} \mathrm{~d} t \\
\leqslant\left|f^{\prime}(a)\right|^{q} \int_{0}^{1}(1-t)^{p}\left[1-\left(1-\frac{t}{2}\right)^{\alpha}\right] \mathrm{d} t \\
\quad+\left|f^{\prime}\left(\frac{b}{m}\right)\right|^{q} \int_{0}^{1} m(1-t)^{p}\left(1-\frac{t}{2}\right)^{\alpha} \mathrm{d} t \\
=\left(\frac{1}{p+1}+\frac{(-1)^{q}}{2^{\alpha}} \beta(\alpha+1, p+1)\right)\left|f^{\prime}(a)\right|^{q} \\
\quad-\frac{m(-1)^{q}}{2^{\alpha}} \beta(\alpha+1, p+1)\left|f^{\prime}\left(\frac{b}{m}\right)\right|^{q} .
\end{array}
$$

Using (31) and (32) in (30), we obtain (29).

Corollary 9 If $g(x)=1$ in Theorem 5, we obtain

$$
\begin{gathered}
\left|\frac{f(a)+f(a+\eta(b, a))}{2}-\frac{1}{\eta(b, a)} \int_{a}^{a+\eta(b, a)} f(x) \mathrm{d} x\right| \\
\leqslant \frac{\eta(b, a)}{4}\left(\frac{q-1}{2 q-p-1}\right)^{(q-1) / q} \\
\times\left\{\left[\left(\frac{1}{p+1}-\frac{1}{2^{\alpha}} \beta(\alpha+1, p+1)\right)\left|f^{\prime}(a)\right|^{q}\right.\right. \\
\left.+\frac{m}{2^{\alpha}} \beta(\alpha+1, p+1)\left|f^{\prime}\left(\frac{b}{m}\right)\right|^{q}\right]^{1 / q}
\end{gathered}
$$

$$
\begin{aligned}
& +\left[\left(\frac{1}{p+1}+\frac{(-1)^{p}}{2^{\alpha}} \beta(\alpha+1, p+1)\right)\left|f^{\prime}(a)\right|^{q}\right. \\
& \left.\left.-\frac{m(-1)^{p}}{2^{\alpha}} \beta(\alpha+1, p+1)\left|f^{\prime}\left(\frac{b}{m}\right)\right|^{q}\right]^{1 / q}\right\}
\end{aligned}
$$

where $p^{-1}+q^{-1}=1$.

Corollary 10 With the same assumptions given in Theorem 5, if $\left|f^{\prime}(x)\right| \leqslant \Upsilon$ on $[a, a+\eta(b, a)]$ with $m=1$, we obtain

$$
\begin{aligned}
& \mid \frac{f(a)+f(a+\eta(b, a))}{2} \int_{a}^{a+\eta(b, a)} g(x) \mathrm{d} x \\
& \quad-\int_{a}^{a+\eta(b, a)} f(x) g(x) \mathrm{d} x \mid \\
& \leqslant \frac{\eta^{2}(b, a)}{2}\left(\frac{q-1}{2 q-p-1}\right)^{(q-1) / q}\left(\frac{1}{p+1}\right)^{1 / q} \Upsilon\|g\|_{\infty}
\end{aligned}
$$

where $p^{-1}+q^{-1}=1$.

Theorem 6 Suppose that all the assumptions of Theorem 4 are satisfied. Then

$$
\begin{gathered}
\mid \frac{f(a)+f(a+\eta(b, a))}{2} \int_{a}^{a+\eta(b, a)} g(x) \mathrm{d} x \\
-\int_{a}^{a+\eta(b, a)} f(x) g(x) \mathrm{d} x \mid \\
\quad \leqslant \frac{\eta^{2}(b, a)}{4}\|g\|_{\infty} \\
\quad \times\left\{\left[\left(\frac{1}{q+1}-\frac{1}{2^{\alpha}} \beta(\alpha+1, q+1)\right)\left|f^{\prime}(a)\right|^{q}\right.\right. \\
\left.\left.+\frac{m}{2^{\alpha}} \beta(\alpha+1, q+1)\left|f^{\prime}\left(\frac{b}{m}\right)\right|\right]^{q}\right]^{1 / q} \\
+\left[\left(\frac{1}{q+1}+\frac{(-1)^{q}}{2^{\alpha}} \beta(\alpha+1, q+1)\right)\left|f^{\prime}(a)\right|^{q}\right. \\
\left.\left.-\frac{m(-1)^{q}}{2^{\alpha}} \beta(\alpha+1, q+1)\left|f^{\prime}\left(\frac{b}{m}\right)\right|^{q}\right]^{1 / q}\right\}
\end{gathered}
$$

where

$$
\beta(x, y)=\int_{0}^{1} t^{x-1}(1-t)^{y-1} \mathrm{~d} t, \quad \forall x, y>0 .
$$

Proof: Using Lemma 1 and Hölder's integral inequality for $p>1$, we obtain

$$
\begin{array}{r}
\mid \frac{f(a)+f(a+\eta(b, a))}{2} \int_{a}^{a+\eta(b, a)} g(x) \mathrm{d} x \\
-\int_{a}^{a+\eta(b, a)} f(x) g(x) \mathrm{d} x \mid
\end{array}
$$




$$
\begin{aligned}
\leqslant & \frac{\eta^{2}(b, a)}{4}\|g\|_{\infty}\left(\int_{0}^{1} 1 \mathrm{~d} t\right)^{1 / p} \\
& \times\left\{\left[\int_{0}^{1}(1-t)^{q}\left|f^{\prime}\left(a+\frac{t}{2} \eta(b, a)\right)\right|^{q} \mathrm{~d} t\right]^{1 / q}\right. \\
& \left.+\left[\int_{0}^{1}(1-t)^{q}\left|f^{\prime}\left(a+\left(1-\frac{t}{2}\right) \eta(b, a)\right)\right|^{q} \mathrm{~d} t\right]^{1 / q}\right\} .
\end{aligned}
$$

Replacing $p$ in (31) and(32) by $q$ and substituting them into (36), we deduce (35).

Corollary 11 If $g(x)=1$ in Theorem 6 , we obtain

$$
\begin{gathered}
\left|\frac{f(a)+f(a+\eta(b, a))}{2}-\frac{1}{\eta(b, a)} \int_{a}^{a+\eta(b, a)} f(x) \mathrm{d} x\right| \\
\leqslant \frac{\eta(b, a)}{4}\left\{\left[\left(\frac{1}{q+1}-\frac{1}{2^{\alpha}} \beta(\alpha+1, q+1)\right)\left|f^{\prime}(a)\right|^{q}\right.\right. \\
\left.\quad+\frac{m}{2^{\alpha}} \beta(\alpha+1, q+1)\left|f^{\prime}\left(\frac{b}{m}\right)\right|^{q}\right]^{1 / q} \\
+\left[\left(\frac{1}{q+1}+\frac{(-1)^{q}}{2^{\alpha}} \beta(\alpha+1, q+1)\right)\left|f^{\prime}(a)\right|^{q}\right. \\
\left.\left.-\frac{m(-1)^{q}}{2^{\alpha}} \beta(\alpha+1, q+1)\left|f^{\prime}\left(\frac{b}{m}\right)\right|^{q}\right]^{1 / q}\right\} .
\end{gathered}
$$

Corollary 12 With the same assumptions given in Theorem 6, if $\left|f^{\prime}(x)\right| \leqslant \Upsilon$ on $[a, a+\eta(b, a)]$ with $m=1$, we obtain

$$
\begin{aligned}
\left.\mid \frac{f(a)+f(a}{2}+\eta(b, a)\right) & \int_{a}^{a+\eta(b, a)} g(x) \mathrm{d} x \\
& -\int_{a}^{a+\eta(b, a)} f(x) g(x) \mathrm{d} x \mid \\
& \leqslant \frac{\eta^{2}(b, a)}{2}\left(\frac{1}{q+1}\right)^{1 / q} \Upsilon\|g\|_{\infty} .
\end{aligned}
$$

Corollary 13 From Corollaries 8, 10 and 12, we have

$$
\begin{aligned}
\mid \frac{f(a)+f(a+\eta(b, a))}{2} \int_{a}^{a+\eta(b, a)} g(x) \mathrm{d} x \\
-\int_{a}^{a+\eta(b, a)} f(x) g(x) \mathrm{d} x \mid \leqslant \min \left\{K_{1}, K_{2}, K_{3}\right\}
\end{aligned}
$$

where

$K_{1}=\frac{\eta^{2}(b, a)}{2}\left(\frac{1}{p+1}\right)^{1 / p} \Upsilon\|g\|_{\infty}$,

$K_{2}=\frac{\eta^{2}(b, a)}{2}\left(\frac{q-1}{2 q-p-1}\right)^{(q-1) / q}\left(\frac{1}{p+1}\right)^{1 / q} \Upsilon\|g\|_{\infty}$, and

$K_{3}=\frac{\eta^{2}(b, a)}{2}\left(\frac{1}{q+1}\right)^{1 / q} \Upsilon\|g\|_{\infty}$.

\section{APPLICATION TO SPECIAL MEANS}

Definition 4 [Ref. 21] A function $M: \mathbb{R}_{+}^{2} \rightarrow \mathbb{R}_{+}$ is called a mean function if it has the following properties.

(i) Homogeneity: $M(a x, a y)=a M(x, y)$ for all $a>0$.

(ii) Symmetry: $M(x, y)=M(y, x)$.

(iii) Reflexivity: $M(x, x)=x$.

(iv) Monotonicity: if $x \leqslant x^{\prime}$ and $y \leqslant y^{\prime}$, then $M(x, y) \leqslant M\left(x^{\prime}, y^{\prime}\right)$.

(v) Internality: $\min \{x, y\} \leqslant M(x, y) \leqslant \max \{x, y\}$.

For arbitrary positive real numbers $a>0$ and $b>0$, we define $A:=A(a, b)=\frac{1}{2}(a+b), G:=$ $G(a, b)=\sqrt{a b}, H:=H(a, b)=2 a b /(a+b)$,

$$
P_{r}:=P_{r}(a, b)=\left(\frac{a^{r}+b^{r}}{2}\right)^{1 / r}, r \geqslant 1
$$$$
I(a, b)= \begin{cases}\frac{1}{e}\left(\frac{b^{b}}{a^{a}}\right)^{1 /(b-a)}, & a \neq b, \\ a, & a=b,\end{cases}
$$

$$
L(a, b)= \begin{cases}\frac{b-a}{\ln b-\ln a}, & a \neq b, \\ a, & a=b,\end{cases}
$$

and

$$
L_{s}(a, b)= \begin{cases}{\left[\frac{b^{s+1}-a^{s+1}}{(s+1)(b-a)}\right]^{\frac{1}{s}},} & s \neq 0,-1, a \neq b, \\ L(a, b), & s=-1, \quad a \neq b, \\ I(a, b), & s=0 \quad a \neq b, \\ a, & a=b .\end{cases}
$$

Clearly, $L_{p}$ is monotonic nondecreasing over $p \in$ $\mathbb{R}$, with $L_{-1}:=L$ and $L_{0}:=I$. In particular, we have $H \leqslant G \leqslant L \leqslant I \leqslant A$.

Now, let $0<a<b$. Suppose that the function $M:=M(a, b):[a+\eta(b, a)] \times[a, a+\eta(b, a)] \rightarrow \mathbb{R}^{+}$, which is one of the abovementioned means. Then one can obtain different inequalities below.

Letting $\eta(b, a)=M(b, a)$ in (18), (23) and (27), one can derive the following significant in- 
equalities.

$$
\begin{aligned}
& \frac{f(a)+f(a+M(b, a))}{2} \\
& -\frac{1}{M(b, a)} \int_{a}^{a+M(b, a)} f(x) \mathrm{d} x \\
& \leqslant \frac{M(b, a)}{8}\left(\left|f^{\prime}(a)\right|+\left|f^{\prime}(b)\right|\right) \\
& \mid \frac{f(a)+f(a+M(b, a))}{2} \\
& -\frac{1}{M(b, a)} \int_{a}^{a+M(b, a)} f(x) \mathrm{d} x \\
& \leqslant \frac{M(b, a)}{4}\left(\frac{q-1}{2 q-1}\right)^{(q-1) / q} \\
& \times \frac{3^{1 / q}+1}{4^{1 / q}}\left(\left|f^{\prime}(a)\right|+\left|f^{\prime}(b)\right|\right)
\end{aligned}
$$

and

$$
\begin{aligned}
& \mid \frac{f(a)+f(a+M(b, a))}{2} \\
& \quad-\frac{1}{M(b, a)} \int_{a}^{a+M(b, a)} f(x) \mathrm{d} x \mid \\
& \quad \leqslant \frac{M(b, a)}{4}\left(\frac{1}{p+1}\right)^{1 / p} \frac{3^{1 / q}+1}{4^{1 / q}}\left(\left|f^{\prime}(a)\right|+\left|f^{\prime}(b)\right|\right) .
\end{aligned}
$$

Letting $M=A, G, H, P_{r}, I, L, L_{s}$ in (39), (40), and (41), one can obtain the required inequalities. The details are left for the reader to explore.

Acknowledgements: This work was partially supported by the National Natural Science Foundation of China under grant No. 61374028.

\section{REFERENCES}

1. Fejér L (1906) Über die Fourierreihen, II. Math Naturwise Anz Ungar Akad Wiss 24, 369-90 [in Hungarian].

2. Weir T, Mond B (1998) Pre-invex functions in multiple objective optimization. J Math Anal Appl 136, 29-38.

3. Latif MA, Shoaib M (2015) Hermite-Hadamard type integral inequalities for differentiable $m$-preinvex and $(\alpha, m)$-preinvex functions. $J$ Egypt Math Soc 23, 236-41.

4. Noor MA (2009) Hadamard integral inequalities for product of two preinvex functions. Nonlin Anal Forum 14, 167-73.
5. Hua J, Xi B-Y, Qi F (2014) Inequalities of HermiteHadamard type involving an $s$-convex function with applications. Appl Math Comput 246, 752-60.

6. Latif MA, Dragomir SS (2015) New inequalities of Hermite-Hadamard and Fejér type via preinvexity. J Comput Anal Appl 19, 725-39.

7. Chen FX, Wu SH (2014) Fejér and HermiteHadamard type inequalities for harmonically convex functions. $J$ Appl Math 2014, 386806.

8. Cortez MV (2016) Fejér type inequalities for $(s, m)$ convex functions in second sense. Appl Math Inform Sci 10, 1-8.

9. Du T-S, Liao J-G, Li Y-J (2016) Properties and integral inequalities of Hadamard-Simpson type for the generalized $(s, m)$-preinvex functions. $J$ Nonlin Sci Appl 9, 3112-26.

10. Du TS, Li YJ, Yang ZQ (2017) A generalization of Simpson's inequality via differentiable mapping using extended $(s, m)$-convex functions. Appl Math Comput 293, 358-69.

11. Fok H, Vong S (2015) Generalizations of some Hermite-Hadamard-type inequalities. Indian $J$ Pure Appl Math 46, 359-70.

12. Hwang S-R, Tseng K-L, Hsu K-C (2013) HermiteHadamard type and Fejér type inequalities for general weights (I). $J$ Inequal Appl 2013, 170.

13. İşcan İ, Kunt M (2016) Hermite-Hadamard-Fejér type inequalities for quasi-geometrically convex functions via fractional integrals. $J$ Math 2016, 6523041.

14. Özdemir ME, Avci M, Set E (2010) On some inequalities of Hermite-Hadamard type via $m$-convexity. Appl Math Lett 23, 1065-70.

15. Sarikaya MZ, Yaldiz H, Erden S (2014) Some inequalities associated with the Hermite-HadamardFejér type for convex function. Math Sci 8, 117-24.

16. Tseng K-L, Yang G-S, Hsu K-C (2011) Some inequalities for differentiable mappings and applications to Fejér inequality and weighted trapezoidal formula. Taiwan J Math 15, 1737-47.

17. Wu SH (2009) On the weighted generalization of the Hermite-Hadamard inequality and its applications. Rocky Mountain J Math 39, 1741-9.

18. Xi B-Y, Qi F, Zhang T-Y (2015) Some inequalities of Hermite-Hadamard type for $m$-harmonicarithmetically convex functions. Sci Asia 41, 357-61.

19. Yang WG (2017) Some new Fejér type inequalities via quantum calculus on finite intervals. Sci Asia 43, 123-34.

20. Barani A, Ghazanfari AG, Dragomir SS (2012) Hermite-Hadamard inequality for functions whose derivatives absolute values are preinvex. $J$ Inequal Appl 2012, 247.

21. Bullen PS (2003) Handbook of Means and Their Inequalities, Kluwer Academic Publishers, Dordreche. 